\title{
Heavy quark production via leptoquarks at a neutrino factory
}

\author{
Ashok Goyal ${ }^{a, 1}$, Poonam Mehta ${ }^{a, 2}$ and Sukanta Dutta ${ }^{b, 3}$ \\ ${ }^{a}$ Department of Physics $\&$ Astrophysics, University of Delhi, Delhi 110 00\%, India \\ ${ }^{b}$ Physics Department, S.G.T.B. Khalsa College, University of Delhi, Delhi 110 00\%, India
}

\begin{abstract}
The proposed neutrino factory (NF) based on a muon storage ring (MSR) is an ideal place to look for heavy quark production via neutral current (NC) and charged current (CC) interactions. In this article, we address the issue of contribution coming from mediating leptoquarks (LQ) in $\nu_{\mu}\left(\bar{\nu}_{e}\right)-\mathrm{N}$ scattering leading to the production of $b(\bar{b})$ at a MSR and investigate the region where LQ interactions are significant in the near-site experiments.
\end{abstract}

Keywords : Leptoquark, Heavy Quark, Muon storage ring, Neutrino Factory.

\section{Introduction}

It is widely believed that the proposed NF based on MSR capable of supplying a well calibrated and intense beam of roughly $\approx 10^{20} \nu_{\mu}\left(\bar{\nu}_{\mu}\right)$ and $\bar{\nu}_{e}\left(\nu_{e}\right)$ per year through $50 \mathrm{GeV}$ muon decays, will open up unprecedented opportunity to reveal the world of neutrino and to provide physical laboratory for testing physics beyond the Standard Model (SM) [1, 2]. Recent strong indications of atmospheric neutrino oscillation $\left(\nu_{\mu} \longrightarrow \nu_{x}\right.$, where $x$ is not $e$ ) [3] have rekindled the interest in accelerator experiments that could study the same range of parameter space. The solar neutrino deficit is interpreted either as matter enhanced Mikhyev-Smirnov-Wolfenstein (MSW) oscillations [4] or as vacuum oscillations [5] that deplete the original $\nu_{e}$ 's, presumably in favour of $\nu_{\mu}$ 's. The role of a NF in determining masses and mixing angles for $\nu_{\mu} \leftrightarrow \nu_{\tau}$ and $\bar{\nu}_{e} \leftrightarrow \bar{\nu}_{\mu}$ oscillations both at short and long baseline experiments has been extensively discussed in the literature. Investigation of physics beyond the SM through certain novel interactions in the neutrino sector, in particular the appearance of $\tau$ and wrong sign $\mu$ signals in new physics scenarios like supersymmetric (SUSY) theories with broken R-parity [6] and theories that allow LQ mediated lepton flavor violating (LFV) interactions [7] have been dealt with in our earlier works $([6],[7])$. With the same motivation to look for the role played by the non-standard interactions at a NF the production of heavy quarks through $\nu_{\mu}$-N scattering in an R-parity violating SUSY theory was investigated recently [8] and it was shown that it is possible to have significant event rates for $b(\bar{b})$ production via both $\mathrm{NC}$ and $\mathrm{CC}$ interactions. We should emphasize here that in SM the production of $b(\bar{b})$ is severely suppressed at tree level. Thus a considerable number of $b(\bar{b})$ or an excess well above the SM rate at a NF would unequivocally imply the existence of non-standard physics in the neutrino sector. In contrast

\footnotetext{
${ }^{1}$ E-mail address: agoyal@ducos.ernet.in

${ }^{2}$ E-mail address: pmehta@physics.du.ac.in, mpoonam@mri.ernet.in

${ }^{3}$ E-mail address: Sukanta.Dutta@cern.ch
} 
to SM, $b$ quark production via non-standard $\nu-N$ scattering processes can take place at the tree level itself via the CC interactions, $\nu_{\mu} \bar{u} \longrightarrow \mu^{-} \bar{b}, \nu_{\mu} \bar{c} \longrightarrow \mu^{-} \bar{b}$ and $\bar{\nu}_{e} u \longrightarrow e^{+} b$, all of which are suppressed in the SM either due to the Cabibbo-Kobayashi-Masakawa (CKM) matrix elements $V_{u b}$ or due to the interaction of $\nu_{\mu}$ with sea quarks present inside the nucleon. The corresponding NC processes $\nu_{\mu} d \longrightarrow \nu_{\mu} b$ and $\bar{\nu}_{e} d \longrightarrow \bar{\nu}_{e} b$ can occur only at one loop level in the SM.

In this context, it is worthwhile to consider theories with leptoquarks which occur naturally in Grand Unified Theories, Superstring inspired $E_{6}$ models and in Technicolor models [9] and study heavy flavour $(b, \bar{b})$ production in scattering of neutrinos on a fixed isonucleon target with LQ as mediators of the interaction. In our earlier work, we have studied the contribution of mediating lepton flavor violating LQ in $\nu_{\mu}\left(\bar{\nu}_{e}\right)$-N scattering leading to an enhanced production of $\tau$ 's and wrong sign $\mu$ 's at MSR and investigated the region where LQ interactions are significant in the near-site and short baseline experiments and we found that one can constrain $L F V$ couplings between the first and third generation, the bounds on which are not generally available. With the same spirit in this present work, we investigate the $b$ quark production in both NC and CC channels through $\nu_{\mu}\left(\bar{\nu}_{e}\right)-\mathrm{N}$ scattering at the NF, mediated by scalar and vector leptoquarks. It is worth mentioning that we consider $\bar{\nu}_{e}$ beam also for production of $b, \bar{b}$ in both the NC and CC channels unlike reference [8]. For the present case since we are interested in new physics effects alone and not the oscillation effects, it is desirable to confine ourselves to near-site experiments where the neutrino detectors are placed at a very short distance (typically $40 \mathrm{~m}$ ) from the storage ring. Here we do not consider the LFV processes. The processes that we consider in this article for the $b, \bar{b}$ production via NC and CC channels are :

$$
\begin{aligned}
\mathrm{NC}: \nu_{\mu} d \longrightarrow \nu_{\mu} b, & \bar{\nu}_{e} d \longrightarrow \bar{\nu}_{e} b \\
\mathrm{CC}: \nu_{\mu} \bar{u} \longrightarrow \mu^{-} \bar{b}, & \bar{\nu}_{e} u \longrightarrow e^{+} b
\end{aligned}
$$

The total number of $b, \bar{b}$ quark production events per year via either CC or NC interactions can be written as

$$
\mathcal{N}_{b, \bar{b}}=\mathcal{N}_{n} \int \frac{d^{2} \sigma_{N C / C C}^{\nu, \bar{\nu}}}{d x d y}\left[\frac{d N_{\nu, \bar{\nu}}}{d E_{\nu_{i}, \bar{\nu}_{i}}}\right] \mathcal{P}_{\text {surv }}\left(\nu_{i}\left(\bar{\nu}_{i}\right) \longrightarrow \nu_{i}\left(\bar{\nu}_{i}\right)\right) d E_{\nu_{i}\left(\bar{\nu}_{i}\right)} q(x) d x d y
$$

where, $\mathcal{N}_{n}$ is the number of nucleons per $\mathrm{kT}$ of the target material ${ }^{1}, x$ and $y$ are the Bjorken scaling variables, q and q' are the quarks in the initial and final states, respectively and $q(x)$ is the quark distribution function. The differential parton level cross-section can be expressed as

$$
\frac{d^{2} \sigma_{N C / C C}^{\nu, \bar{\nu}}}{d x d y}=\left[\frac{d^{2} \sigma_{N C / C C}^{\nu, \bar{\nu}}}{d x^{\prime} d y^{\prime}}\right] \times \frac{\partial\left(x^{\prime}, y^{\prime}\right)}{\partial(x, y)}=\left[\frac{\left|\mathcal{M}\left(x^{\prime}, y^{\prime}\right)\right|_{N C / C C}^{2}}{32 \pi \hat{S}}\right]
$$

where $y^{\prime}=-\hat{t} / \hat{S}=Q^{2} /\left(2 M E_{\nu} x^{\prime}\right), x^{\prime}$ is the slow rescaling variable ${ }^{2}$ that arises due to the mass shell constraint of the heavy quark produced in the final state,

$$
x^{\prime}=\frac{Q^{2}+m_{Q}^{2}}{2 M \nu}=x+\frac{m_{Q}^{2}}{2 M E_{\nu} y} \quad \text { Therefore } \quad \frac{\partial\left(x^{\prime}, y^{\prime}\right)}{\partial(x, y)}=1 .
$$

with $M$ being the nucleon mass, $E_{\nu}$ being the neutrino energy and $\nu=E_{\nu_{l}}-E_{l^{-}}\left(E_{\bar{\nu}_{l}}-E_{l^{+}}\right)$. $\hat{S}$ is the parton level CM energy and $\left[\frac{d N_{\nu, \bar{\nu}}}{d E_{\nu_{i}, \bar{\nu}_{i}}}\right]$ is the differential $\nu(\bar{\nu})$ flux. The survival

\footnotetext{
${ }^{1} \mathcal{N}_{n}=6.023 \times 10^{32}$ for a target of mass $1 \mathrm{kT}$.

${ }^{2}$ For production of a heavy quark from a light quark, the heavy quark mass modifies the scaling variable of the quark distribution. $x^{\prime}$ is the quark momentum fraction appropriate to absorb the virtual $W$ described by $\nu$ and $Q^{2}$.
} 
probability of a particular neutrino flavour $(i)$ is given by $\mathcal{P}_{\text {surv }}\left(\nu_{i} \rightarrow \nu_{i}\right)=1-\mathcal{P}_{\text {osc }}\left(\nu_{i} \rightarrow \nu_{j}\right)$ where $\mathrm{j}$ takes all possible values, $j=e, \mu, \tau$ but $j \neq i^{3}$.

The effective Lagrangian with the most general dimensionless, $S U(3)_{c} \mathrm{X} S U(2)_{L} \mathrm{XU}(1)_{Y}$ invariant couplings of scalar and vector LQ satisfying baryon $(B)$ and lepton number $(L)$ conservation (suppressing colour, weak isospin and generation (flavour) indices ) is given [10] by:

$$
\begin{aligned}
\mathcal{L} & =\mathcal{L}_{|F|=2}+\mathcal{L}_{|F|=0} \quad \text { where } \\
\mathcal{L}_{|F|=2} & =\left[g_{1 L} \bar{q}_{L}^{c} i \tau_{2} l_{L}+g_{1 R} \bar{u}_{R}^{c} e_{R}\right] S_{1}+\tilde{g}_{1 R} \bar{d}_{R}^{c} e_{R} \tilde{S}_{1}+g_{3 L} \bar{q}_{L}^{c} i \tau_{2} \vec{\tau} l_{L} \vec{S}_{3} \\
& +\left[g_{2 L} \bar{d}_{R}^{c} \gamma^{\mu} l_{L}+g_{2 R} \bar{q}_{L}^{c} \gamma^{\mu} e_{R}\right] V_{2 \mu}+\tilde{g}_{2 L} \bar{u}_{R}^{c} \gamma^{\mu} l_{L} \tilde{V}_{2 \mu}+\text { c.c. }, \\
\mathcal{L}_{|F|=0} & =\left[h_{2 L} \bar{u}_{R} l_{L}+h_{2 R} \bar{q}_{L} i \tau_{2} e_{R}\right] R_{2}+\tilde{h}_{2 L} \bar{d}_{R} l_{L} \tilde{R}_{2}+\tilde{h}_{1 R} \bar{u}_{R} \gamma^{\mu} e_{R} \tilde{U}_{1 \mu} \\
& +\left[h_{1 L} \bar{q}_{L} \gamma^{\mu} l_{L}+h_{1 R} \bar{d}_{R} \gamma^{\mu} e_{R}\right] U_{1 \mu}+h_{3 L} \bar{q}_{L} \vec{\tau} \gamma^{\mu} l_{L} U_{3 \mu}+\text { c.c. }
\end{aligned}
$$

where $q_{L}, l_{L}$ are the left-handed quarks and lepton doublets and $e_{R}, d_{R}, u_{R}$ are the righthanded charged leptons, down- and up-quark singlets respectively . The Scalar (i.e. $S_{1}, \tilde{S}_{1}$, $S_{3}$ ) and Vector (i.e. $V_{2}, \tilde{V}_{2}$ ) LQ carry fermion number $\mathrm{F}=3 \mathrm{~B}+\mathrm{L}=-2$, while the Scalar (i.e. $R_{2}, \tilde{R}_{2}$ ) and Vector (i.e. $\left.U_{1}, \tilde{U}_{1}, U_{3}\right)$ LQ have $\mathrm{F}=0$.

Numerous phenomenological studies have been made in order to derive bounds and put stringent constraints on LQ couplings particularly from low energy FCNC processes [11] that are generated by scalar and vector LQ interactions. Direct experimental searches for leptoquarks have also been carried out at the e-p collider and bounds obtained $[11,12]$ and in particular bounds obtained from $B$ meson decays $\left(B \longrightarrow l^{+} l^{-} X\right.$, where $\left.l^{+} l^{-}=\mu^{+} \mu^{-}, e^{+} e^{-}\right)$ and also bounds derived from meson-antimeson $(B \bar{B})$ mixing would have direct bearing on the processes considered here. This is because low energy limit puts stringent bound on effective four-fermion interactions involving two leptons and two quarks and since at the NF the centre of mass energy in collision is low enough, we can consider the neutrino-quark interaction as an effective four-fermion interaction. The bounds on effective couplings used in this paper are the LQ couplings over mass squared of the LQ and are derived on the assumption that individual leptoquark coupling contribution to the branching ratio does not exceed the experimental upper limits and in the branching ratios only one leptoquark coupling is considered by switching off all the other couplings. All couplings are considered to be real and combinations of left and right chirality coupling are not considered. This article is outlined as follows. We discuss the $b(\bar{b})$ production through $\nu(\bar{\nu})-\mathrm{N}$ interactions via $\mathrm{NC}$ and $\mathrm{CC}$ channels in section 2 and 3 respectively and give the plots of event rate versus muon beam energy. In section 4 we outline the conclusions drawn from our results.

\section{$2 \mathrm{~b}(\overline{\mathrm{b}})$ Production Via NC Processes:}

Let us first consider the possible NC processes that can lead to $b / \bar{b}$ in the final state. There is no $\mathrm{SM}$ tree level process in the $\mathrm{NC}$ channel as NC processes leading to $b / \bar{b}$ can only occur at one loop level in the SM. However, there can be two possible non-standard tree level NC processes that can lead to the production of $b / \bar{b}$ in the final state, due to the presence of both $\nu$ and $\bar{\nu}$ of different flavors from $\mu$ decay, viz $\mu^{-} \longrightarrow e^{-} \nu_{\mu} \overline{\nu_{e}}$

\footnotetext{
${ }^{3}$ For two flavour oscillation case, $\mathcal{P}_{\text {osc }}\left(\nu_{i} \rightarrow \nu_{j}\right)=\sin ^{2} 2 \theta_{m} \sin ^{2}\left[1.27 \Delta m^{2}\left[\mathrm{eV}^{2}\right] \frac{L[\mathrm{~km}]}{E_{\nu}[\mathrm{GeV}]}\right]$, where, $L$ is the baseline length, $E_{\nu}$ is the neutrino energy, $\Delta m^{2}$ is the mass-squared difference between the corresponding physical states, and $\theta_{m}$ is mixing angle between flavours.
} 
1. $\nu_{\mu}+d \longrightarrow \nu_{\mu}+b$

2. $\bar{\nu}_{e}+d \longrightarrow \bar{\nu}_{e}+b$

For the two NC processes mentioned above, we have both s- and u-channel diagrams arising from the relevant interaction terms in the effective LQ lagrangian. For the first process, $\nu_{\mu}+d \longrightarrow \nu_{\mu}+b$ ( shown in figure 1 ), there are two possible u-channel diagrams mediated by LQs $\left(\tilde{R}^{\dagger}, U^{\dagger}\right)$ carrying $|\mathrm{F}|=0$ and charge $=1 / 3$, while there are three possible s-channel diagrams that are mediated by LQs $\left(S^{\dagger}, V^{\dagger}\right)$ carrying $|\mathrm{F}|=2$ and charge $=-1 / 3$. For the second process, $\bar{\nu}_{e}+d \longrightarrow \bar{\nu}_{e}+b$ ( shown in figure 2 ), the two possible s-channel diagrams are mediated by LQs $(\tilde{R}, U)$ carrying $|\mathrm{F}|=0$ and charge $=-1 / 3$, while the three possible u-channel diagrams are mediated by LQs $(S, V)$ carrying $|\mathrm{F}|=2$ and charge $=1 / 3$.

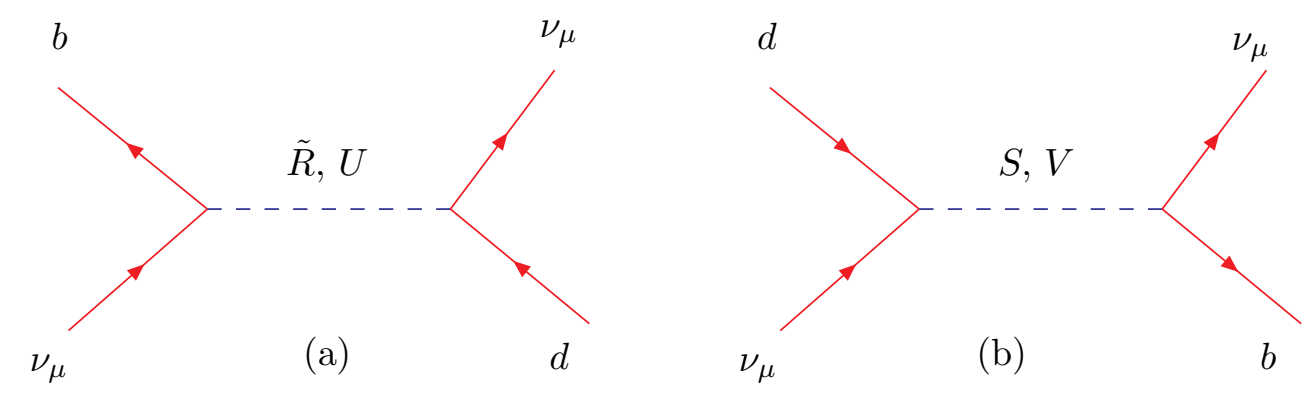

Figure 1: $b$ production via $N C$ process $\left(\nu_{\mu}+d \longrightarrow \nu_{\mu}+b\right)$ from scalar $\&$ vector $L Q$ : (a) $u$ channel process corresponding to $|\mathrm{F}|=0$ LQ and (b) s-channel process corresponding to $|F|=2$ $L Q$.

We first consider the production of "b" from $\nu_{\mu}$ (obtained from $\mu^{-}$decay) interactions with nucleon via NC u-channel processes for $|F|=0$ case ( figure 1(a)) and NC s-channel processes for $|F|=2$ case ( figure 1(b)). There are in all two diagrams contributing to production of $\mathrm{b}$ via $\left(\nu_{\mu}+d \longrightarrow \nu_{\mu}+b\right)$ in the $\mathrm{u}$-channel (figure $1(\mathrm{a})$ ), one mediated by the charge $=1 / 3$, scalar LQ $\left({R_{2}^{-1 / 2}}^{\dagger}\right)$ carrying $T_{3}=-1 / 2$ and the other one by a vector LQ $\left(U_{3 \mu}^{-\dagger}\right)$ with $T_{3}=-1$, where $T_{3}$ is the weak isospin. The matrix element squared for 2 diagrams contributing to the $\mathrm{u}$-channel $\mathrm{NC}$ process is $\left|\mathcal{M}_{L Q}^{u-\operatorname{chann}}\left(\nu_{\mu} d \longrightarrow \nu_{\mu} b\right)\right|^{2}=\left[\hat{u}\left(\hat{u}-m_{b}^{2}\right)\right]\left[\frac{\left|\tilde{h_{2 L}} \tilde{h_{2 L}}\right|^{2}}{\left(\hat{u}-M_{R_{2}^{2}}^{2} \tilde{c}\right)^{2}}\right]+\left[4 \hat{s}\left(\hat{s}-m_{b}^{2}\right)\right]\left[\frac{\left|\sqrt{2} h_{3 L} \sqrt{2} h_{3 L}\right|^{2}}{\left(\hat{u}-M_{U_{3 \mu}^{-}}^{2}\right)^{2}}\right]$

where, the Mandelstam variables at the parton level are given by $\hat{s}=\left(p_{\nu_{\mu}}+p_{d}\right)^{2}, \hat{t}=$ $\left(p_{\nu_{\mu}}(\text { initial })-p_{\nu_{\mu}}(\text { final })\right)^{2}$ and $\hat{u}=\left(p_{\nu_{\mu}}-p_{b}\right)^{2}$, with $p_{i}$ denoting the four momentum of the $i^{\text {th }}$ particle.

In the s-channel, two diagrams are mediated by charge $=-1 / 3$, scalar LQs $\left(S_{1}^{\dagger}, S_{3}^{0^{\dagger}}\right)$ with $T_{3}=0$, while one is mediated by a vector LQ $\left(V_{2 \mu}^{-1 / 2^{\dagger}}\right)$ with $T_{3}=-1 / 2$ ( figure $1(\mathrm{~b})$ ). The matrix element squared for all the 3 diagrams contributing to the NC s-channel process is

$$
\left|\mathcal{M}_{L Q}^{s-\operatorname{chann}}\left(\nu_{\mu} d \longrightarrow \nu_{\mu} b\right)\right|^{2}=\left[\hat{s}\left(\hat{s}-m_{b}^{2}\right)\right]\left[\frac{\left|g_{1 L} g_{1 L}\right|^{2}}{\left(\hat{s}-M_{S_{1}}^{2}\right)^{2}}+\frac{\left|g_{3 L} g_{3 L}\right|^{2}}{\left(\hat{s}-M_{S_{3}^{0}}^{2}\right)^{2}}\right.
$$




$$
\left.+2 \frac{\left|g_{1 L} g_{3 L}\right|^{2}}{\left(\hat{s}-M_{S_{1}}^{2}\right)\left(\hat{s}-M_{S_{3}^{0}}^{2}\right)}\right]+\left[4 \hat{u}\left(\hat{u}-m_{b}^{2}\right)\right]\left[\frac{\left|g_{2 L} g_{2 L}\right|^{2}}{\left(\hat{s}-M_{V_{2 \mu}^{-1 / 2}}^{2}\right)^{2}}\right]
$$

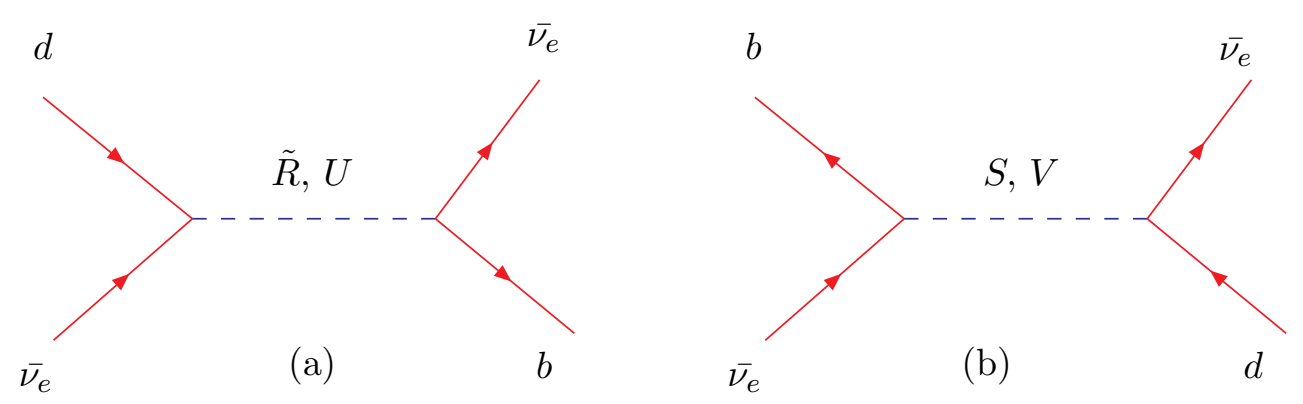

Figure 2: $b$ production via NC process $\left(\bar{\nu}_{e}+d \longrightarrow \bar{\nu}_{e}+b\right)$ from scalar $\&$ vector $L Q:(a)$ s-channel process corresponding to $|\mathrm{F}|=0 L Q$ and $(b)$ u-channel process corresponding to $|F|=2 L Q$.

Next we consider the production of "b" from $\overline{\nu_{e}}$ (also obtained from the $\mu^{-}$decay) through interactions with nucleon via NC s-channel process for $|F|=0$ case ( figure 2(a)) and NC u-channel process for $|F|=2$ case ( figure 2(b)). There are in all two diagrams contributing to production of $\mathrm{b}$ via $\left(\overline{\nu_{e}}+d \longrightarrow \overline{\nu_{e}}+b\right)$ in the s-channel ( figure $2(\mathrm{a})$ ), one mediated by the charge $=-1 / 3$, scalar LQ $\left(\tilde{R}_{2}^{-1 / 2}\right)$ with $T_{3}=-1 / 2$ and while the other one by a vector LQ $\left(U_{3 \mu}^{-}\right)$with $T_{3}=-1$. The matrix element squared for the 2 diagrams contributing to the $\mathrm{NC}$ s-channel process is

$\left|\mathcal{M}_{L Q}^{s-\operatorname{chann}}\left(\overline{\nu_{e}} d \longrightarrow \overline{\nu_{e}} b\right)\right|^{2}=\left[\hat{s}\left(\hat{s}-m_{b}^{2}\right)\right]\left[\frac{\left|\tilde{h_{2 L}} \tilde{h_{2 L}}\right|^{2}}{\left(\hat{s}-M_{R_{2}^{-1 / 2}}^{2}\right)^{2}}\right]+\left[4 \hat{u}\left(\hat{u}-m_{b}^{2}\right)\right]\left[\frac{\left|\sqrt{2} h_{3 L} \sqrt{2} h_{3 L}\right|^{2}}{\left(\hat{s}-M_{U_{3 \mu}^{-}}^{2}\right)^{2}}\right]$

where, the Mandelstam variables at the parton level are given by $\hat{s}=\left(p_{\overline{\nu_{e}}}+p_{d}\right)^{2}, \hat{t}=$ $\left(p_{\overline{\nu_{e}}}(\text { initial })-p_{\overline{\nu_{e}}}(\text { final })\right)^{2}$ and $\hat{u}=\left(p_{\overline{\nu_{e}}}-p_{b}\right)^{2}$.

In the u-channel, two diagrams are mediated by the charge $=1 / 3$, scalar LQs $\left(S_{1}, S_{3}^{0}\right)$ with $T_{3}=0$ and one is mediated by a vector $\mathrm{LQ}\left(V_{2 \mu}^{-1 / 2}\right)$ with $T_{3}=-1 / 2$ ( figure $\left.2(\mathrm{~b})\right)$. The matrix element squared for all 3 diagrams contributing to the $\mathrm{NC}$ u-channel process is

$$
\begin{aligned}
& \left|\mathcal{M}_{L Q}^{u-\operatorname{chann}}\left(\overline{\nu_{e}} d \longrightarrow \overline{\nu_{e}} b\right)\right|^{2}=\left[\hat{u}\left(\hat{u}-m_{b}^{2}\right)\right]\left[\frac{\left|g_{1 L} g_{1 L}\right|^{2}}{\left(\hat{u}-M_{S_{1}}^{2}\right)^{2}}+\frac{\left|g_{3 L} g_{3 L}\right|^{2}}{\left(\hat{u}-M_{S_{3}^{0}}^{2}\right)^{2}}\right. \\
& \left.+2 \frac{\left|g_{1 L} g_{3 L}\right|^{2}}{\left(\hat{u}-M_{S_{1}}^{2}\right)\left(\hat{u}-M_{S_{3}^{0}}^{2}\right)}\right]+\left[4 \hat{s}\left(\hat{s}-m_{b}^{2}\right)\right]\left[\frac{\left|g_{2 L} g_{2 L}\right|^{2}}{\left(\hat{u}-M_{V_{2 \mu}^{-1 / 2}}^{2}\right)^{2}}\right]
\end{aligned}
$$

Having said all about the relevant NC diagrams leading to b-production, we now focus on the details that we use in order to compute the number of events for $b / \bar{b}$ via NC channel and demonstrate their behaviour as a function of muon energy ranging from 0 upto $250 \mathrm{GeV}$. We consider the contribution from LQ carrying different fermion numbers separately, which 


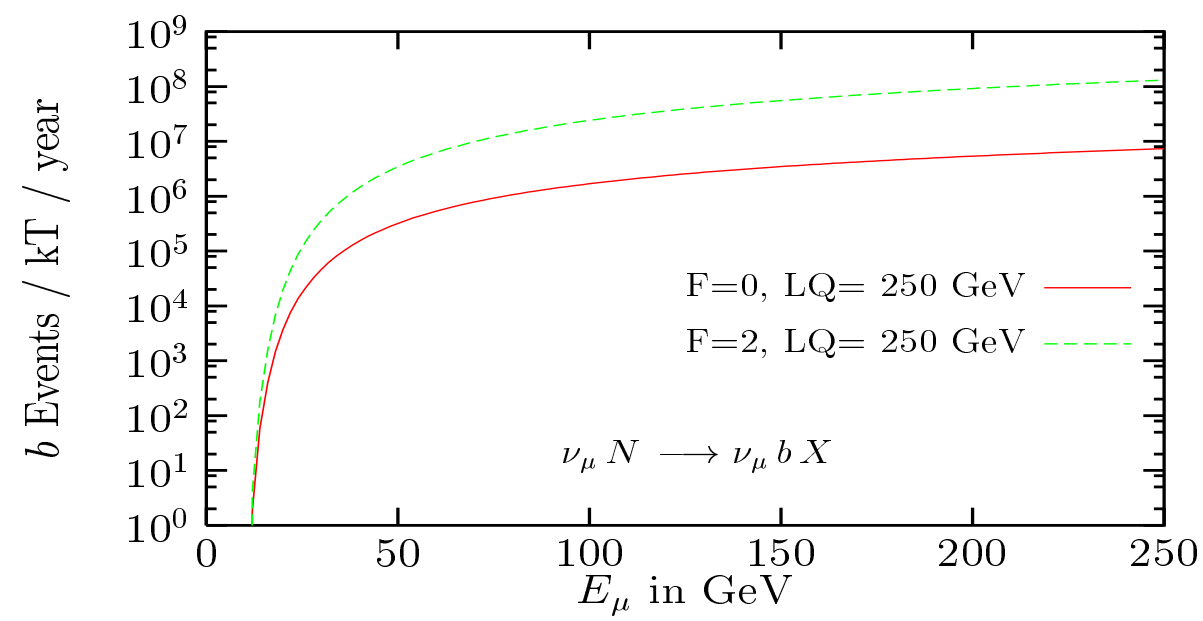

Figure 3: Variation of b-events ( from $L Q$ ) for a $1 k T$ detector and $L Q$ mass 250 GeV with muon beam energy for a baseline length 40 meters and sample detector area $0.025 \mathrm{~m}^{2}$

essentially means that either all the h's or all the g's contributing to a given process, are non-zero at a time. For simplicity, we take the masses of scalar and vector LQs for both $\mathrm{F}=0$ and $|\mathrm{F}|=2$ to be equal $(=250 \mathrm{GeV})$. As in our earlier works ( $[6],[7]$ ), we have used CTEQ4LQ Parton Distribution Functions [13] in order to compute the events. There is however significant suppression in phase space due to the production of massive ' $b$ ' quark. In our calculation we have not imposed any event selection cuts. Using events selection cuts for detail analysis as given in reference [2] will further scale down the contribution. We have considered a detector with a sample area of $0.025 \mathrm{~m}^{2}$ [14] and placed at $40 \mathrm{~m}$ from the storage ring. Regarding the bounds on LQ couplings, we have used model independent constraints on the couplings to $b$ quarks of $B$ and $L$ conserving LQs as discussed in [11] where it is shown that one can constrain the generation dependent LQ couplings to $b$ quarks from the upper bounds on the flavour-changing decays $B \longrightarrow l^{+} l^{-} X$ (where $l^{+} l^{-}=\mu^{+} \mu^{-}, e^{+} e^{-}$), the CKM matrix element $V_{u b}$ and from meson - antimeson $(B \bar{B})$ mixing and obtain some of the best bounds for the processes of our interest. All the bounds on couplings that we have used for calculation of event rates are listed in table 1 . Since the bounds on the couplings $h_{2 L} \& g_{1 R}$ are not available from reference [11], we take them to be the same as bounds on couplings $h_{2 R} \& g_{1 L}$ (which are the opposite chirality counterparts of $h_{2 L} \& g_{1 R}$ respectively). We make some simplifying assumptions like the product of couplings of different chirality is obtained from the squares of the couplings of individual chiralities. We extract bounds relevant to $\left(\nu_{\mu} d\right)\left(\nu_{\mu} b\right)$ vertex from the bounds for $(21)(23)$ generation of quark-lepton pair, while for the vertex $\left(\bar{\nu}_{e} d\right)\left(\bar{\nu}_{e} b\right)$, we use the bounds for the (11)(13) generation indices relevant to the process. These bounds are derived from semileptonic inclusive B decays. The latest bounds coming from BABAR and BELLE experiments [15] however are not relevant for the processes considered here except for the bound on $V_{u b}$ which does not make any significant change in the couplings. In figure 3 and 4, we have plotted the b-quark production rate as a function of muon beam energy for $\nu_{\mu}-\mathrm{N}$ and $\bar{\nu}_{e}-\mathrm{N}$ scattering processes respectively. 


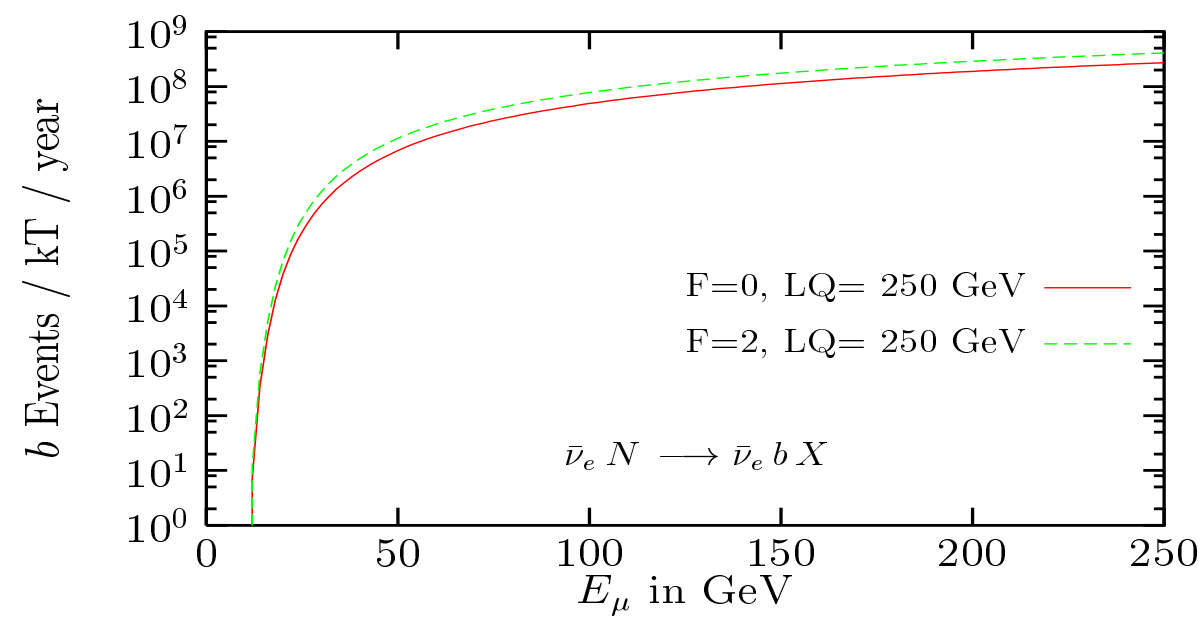

Figure 4: Variation of b-events ( from $L Q$ ) for a $1 k T$ detector and $L Q$ mass 250 GeV with muon beam energy for a baseline length 40 meters and sample detector area $0.025 \mathrm{~m}^{2}$

\begin{tabular}{|l|l|l|l|l|l|l|l|l|l|l|}
\hline \hline$(\mathrm{lq})(\mathrm{lq})$ & $h_{1 L}$ & $h_{1 R}$ & $h_{2 L}$ & $h_{2 R}$ & $h_{3 L}$ & $g_{1 L}$ & $g_{1 R}$ & $g_{2 L}$ & $g_{2 R}$ & $g_{3 L}$ \\
\hline \hline$(11)(13)$ & .002 & .003 & - & .006 & .002 & .004 & - & .003 & .003 & .004 \\
\hline \hline$(21)(23)$ & .0004 & .0004 & - & .0008 & .0004 & .004 & - & .0004 & .0004 & .0004 \\
\hline \hline
\end{tabular}

Table 1: The best bounds on all relevant products of couplings (from $\mathrm{B}$ decays and $B \bar{B}$ mixing) taken from table 15 of the reference [11] by S. Davidson et al. ). All the bounds are multiplied by $\left(m_{L Q} /[100 \mathrm{GeV}]\right)^{2}$.

\section{3 b $(\bar{b})$ Production Via CC Processes:}

As discussed above the production of $\bar{b}$ or $b$ in the final state through CC interaction can also occur in the SM at the tree level in contrast to the NC case where SM contributes only at the one loop level. The SM cross-sections for the CC processes $\nu_{\mu}+\bar{u} \longrightarrow \mu^{-}+\bar{b}$ and $\bar{\nu}_{e}+u \longrightarrow e^{+}+b$ are given by

$$
\begin{aligned}
\frac{d^{2} \sigma}{d x d y}\left(\nu_{\mu} N \longrightarrow \mu^{-} \bar{b} X\right) & =\frac{G_{F}^{2} S}{\pi}\left(\frac{M_{W}^{2}}{M_{W}^{2}+Q^{2}}\right)^{2}\left(x^{\prime}-x^{\prime} y^{\prime}-\frac{m_{b}^{2}}{S}\right)\left(1-y^{\prime}\right) \bar{u}\left(x^{\prime}\right)\left|V_{u b}\right|^{2} \\
\frac{d^{2} \sigma}{d x d y}\left(\bar{\nu}_{e} N \longrightarrow e^{+} b X\right) & =\frac{G_{F}^{2} S}{\pi}\left(\frac{M_{W}^{2}}{M_{W}^{2}+Q^{2}}\right)^{2}\left(x^{\prime}-x^{\prime} y^{\prime}-\frac{m_{b}^{2}}{S}\right)\left(1-y^{\prime}\right) u\left(x^{\prime}\right)\left|V_{u b}\right|^{2}
\end{aligned}
$$

Here we have the advantage of having the SM rates as benchmark against which to the compare the rates obtained via LQ. $\bar{u}\left(x^{\prime}\right)$ and $u\left(x^{\prime}\right)$ are the distribution functions of up-type antiquark and quark respectively.

For the CC processes mentioned above, we can have both s- and u-channel diagrams arising from the relevant interaction terms in the effective LQ Lagrangian, as for the case of NC processes. For the first process, $\nu_{\mu}+\bar{u} \longrightarrow \mu^{-}+\bar{b}$ ( as shown in figure 3 ), there are 4 possible s-channel diagrams mediated by LQs $\left(R^{\dagger}, U^{\dagger}\right)$ carrying $|\mathrm{F}|=0$ and charge $=-2 / 3$. Also there are 4 possible $\mathrm{u}$-channel diagrams that are mediated by LQs $\left(S^{\dagger}, V^{\dagger}\right)$ carrying $|\mathrm{F}|=2$ and charge $=-1 / 3$. For the second process, $\bar{\nu}_{e}+u \longrightarrow e^{+}+b$ ( as shown in figure 4 ), the 4 


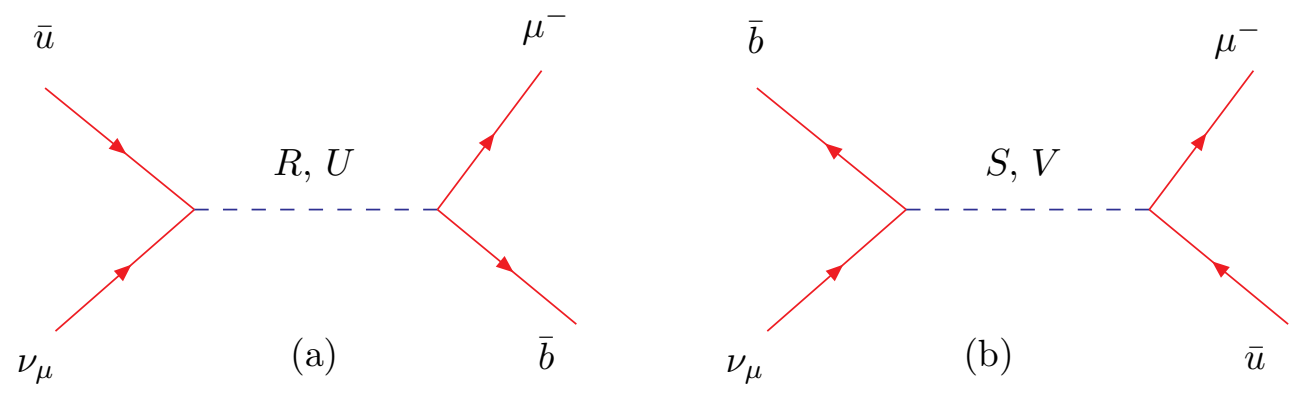

Figure 5: b production via CC process $\left(\nu_{\mu}+\bar{u} \longrightarrow \mu^{-}+\bar{b}\right)$ from scalar 6 vector $L Q$ : (a) s-channel diagram corresponding to $|\mathrm{F}|=0 L Q$ and (b) u-channel diagram corresponding to $|\mathrm{F}|=2 L Q$.

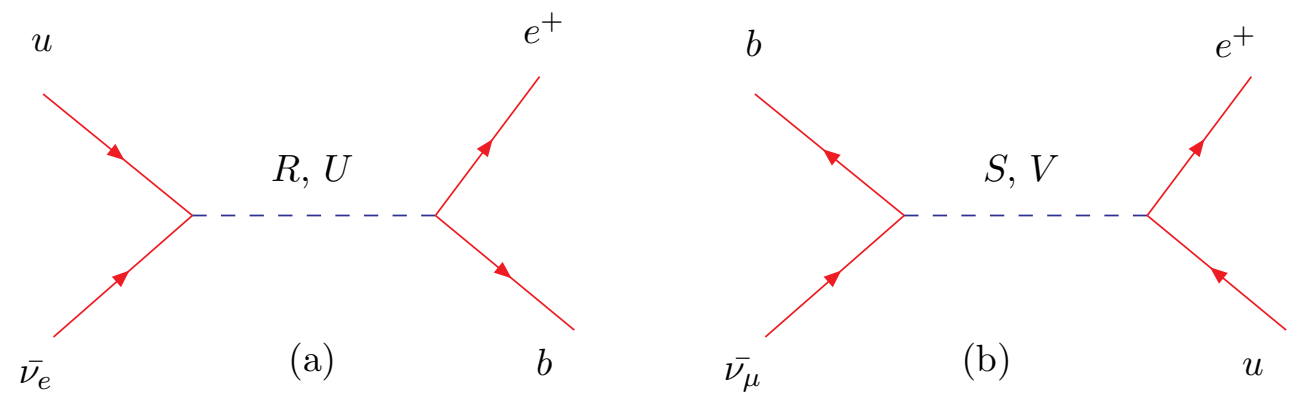

Figure 6: $b$ production via CC process $\left(\bar{\nu}_{e}+u \longrightarrow e^{+}+b\right)$ from scalar $\&$ vector $L Q:(a)$ s-channel diagram corresponding to $|\mathrm{F}|=0 L Q$ and (b) u-channel diagram corresponding to $|\mathrm{F}|=2 L Q$.

possible s-channel diagrams are mediated by LQs $(R, U)$ carrying $|\mathrm{F}|=0$ and charge $=2 / 3$, while the 4 possible u-channel diagrams are mediated by LQs $(S, V)$ carrying $|\mathrm{F}|=2$ and charge $=1 / 3$, respectively. We first consider the production of " $\bar{b}$ " from $\nu_{\mu}$ (obtained from $\mu^{-}$ decay) through interactions with nucleon via CC s-channel process for the $|F|=0$ case ( figure $5(\mathrm{a})$ ) and $\mathrm{CC}$ u-channel process for the $|F|=2$ case ( figure $5(\mathrm{~b})$ ).

There are in all 4 diagrams contributing to production of $\bar{b}$ via $\left(\nu_{\mu}+\bar{u} \longrightarrow \mu^{-}+\bar{b}\right)$ in the s-channel ( figure 5(a) ), one mediated by the charge $=-2 / 3$, scalar LQ $\left(R_{2}^{-1 / 2^{\dagger}}\right.$ ) with $T_{3}=-1 / 2$ and the other three by vector LQs $\left(U_{1 \mu}{ }^{\dagger}, U_{1 \mu}{ }^{\dagger}, U_{3 \mu}^{0}{ }^{\dagger}\right)$ with $T_{3}=-1$. The matrix element squared for all 4 diagrams contributing to the $\mathrm{CC}$ s-channel process is

$$
\begin{gathered}
\left|\mathcal{M}_{L Q}^{s-\operatorname{chann}}\left(\nu_{\mu} \bar{u} \longrightarrow \mu^{-} \bar{b}\right)\right|^{2}=\left[\hat{s}\left(\hat{s}-m_{b}^{2}\right)\right]\left[\frac{\left|h_{2 L} h_{2 R}\right|^{2}}{\left(\hat{s}-M_{R_{2}^{-1 / 2}}^{2}\right)^{2}}\right]+\left[4 \hat{u}\left(\hat{u}-m_{b}^{2}\right)\right]\left[\frac{\left|h_{1 L} h_{1 L}\right|^{2}}{\left(\hat{s}-M_{U_{1 \mu}}^{2}\right)^{2}}\right. \\
\left.+\frac{\left|h_{3 L} h_{3 L}\right|^{2}}{\left(\hat{s}-M_{U_{3 \mu}^{0}}^{2}\right)^{2}}-2 \frac{\left|h_{1 L} h_{3 L}\right|^{2}}{\left(\hat{s}-M_{U_{1 \mu}}^{2}\right)\left(\hat{s}-M_{U_{3 \mu}^{0}}^{2}\right)}\right]+\left[4(\hat{s}+\hat{u})\left(\hat{s}+\hat{u}-m_{b}^{2}\right)\right]\left[\frac{\left|h_{1 L} h_{1 R}\right|^{2}}{\left(\hat{s}-M_{U_{1 \mu}}^{2}\right)^{2}}\right]
\end{gathered}
$$

where, the Mandelstam variables at the parton level are given by $\hat{s}=\left(p_{\nu_{\mu}}+p_{\bar{u}}\right)^{2}, \hat{t}=\left(p_{\nu_{\mu}}-\right.$ $\left.p_{\mu^{-}}\right)^{2}$ and $\hat{u}=\left(p_{\nu_{\mu}}-p_{\bar{b}}\right)^{2}$.

In the u-channel, 3 diagrams are mediated by the charge $=-1 / 3$, scalar LQs $\left(S_{1}^{\dagger}, S_{1}^{\dagger}, S_{3}^{0^{\dagger}}\right)$ with $T_{3}=0$ and one is mediated by a vector LQ $\left(V_{2 \mu}^{-1 /{ }^{\dagger}}\right)$ with $T_{3}=-1 / 2($ figure $5(\mathrm{~b}))$. The 


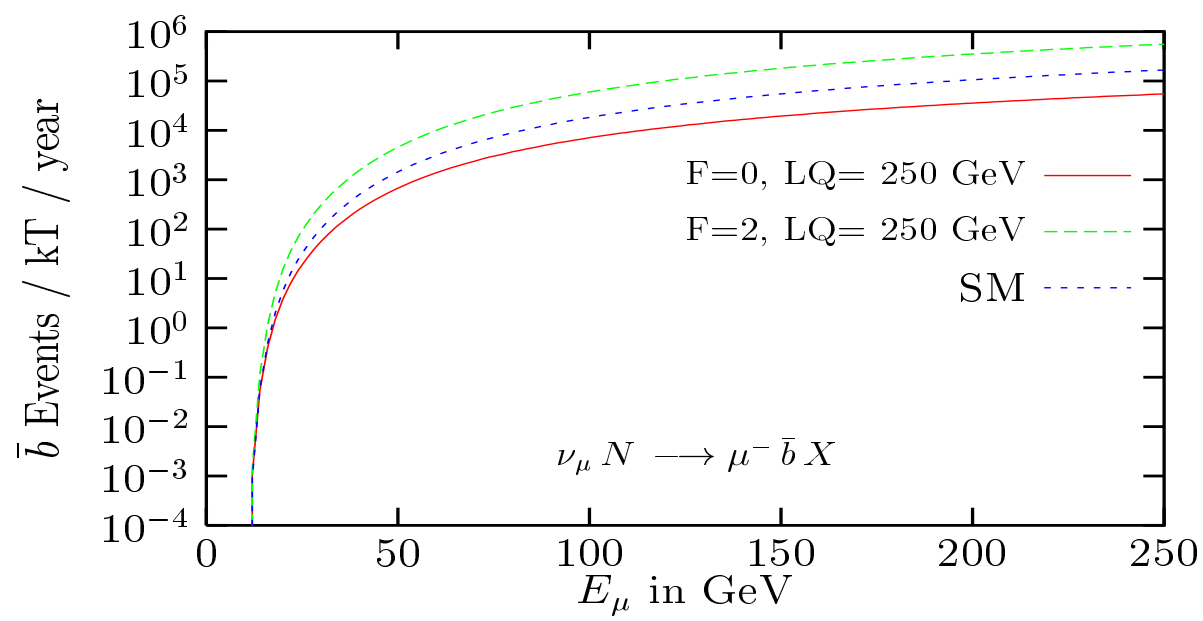

Figure 7: Variation of $\bar{b}$-events (from $S M$ and $L Q$ ) for a $1 k T$ detector and $L Q$ mass $250 \mathrm{GeV}$ with muon beam energy for a baseline length 40 meters and sample detector area $0.025 \mathrm{~m}^{2}$

matrix element squared for all 4 diagrams contributing to the $\mathrm{CC}$ u-channel process is

$$
\begin{aligned}
\mid \mathcal{M}_{L Q}^{u-\operatorname{chann}\left(\nu_{\mu} \bar{u}\right.} & \left.\longrightarrow \mu^{-} \bar{b}\right)\left.\right|^{2}=\left[\hat{u}\left(\hat{u}-m_{b}^{2}\right)\right]\left[\frac{\left|g_{1 L} g_{1 L}\right|^{2}}{\left(\hat{u}-M_{S_{1}}^{2}\right)^{2}}+\frac{\left|g_{1 L} g_{1 R}\right|^{2}}{\left(\hat{u}-M_{S_{1}}^{2}\right)^{2}}+\frac{\left|g_{3 L} g_{3 L}\right|^{2}}{\left(\hat{u}-M_{S_{3}^{0}}^{2}\right)^{2}}\right. \\
& \left.-2 \frac{\left|g_{1 L} g_{3 L}\right|^{2}}{\left(\hat{u}-M_{S_{1}}^{2}\right)\left(\hat{u}-M_{S_{3}^{0}}^{2}\right)}\right]+\left[4(\hat{s}+\hat{u})\left(\hat{s}+\hat{u}-m_{b}^{2}\right)\right]\left[\frac{\left|g_{2 L} g_{2 L}\right|^{2}}{\left(\hat{u}-M_{V_{2 \mu}^{-1 / 2}}^{2}\right)^{2}}\right]
\end{aligned}
$$

Next we consider the production of "b" from $\overline{\nu_{e}}$ (also obtained from the $\mu^{-}$decay) through interactions with nucleon via CC s-channel process for $|F|=0$ case ( figure 6(a)) and CC u-channel process for $|F|=2$ case ( figure 6(b) ). There are in all 4 diagrams contributing to production of $\mathrm{b}$ via $\left(\overline{\nu_{e}}+u \longrightarrow e^{+}+b\right)$ in the s-channel ( figure $6(\mathrm{a})$ ), one mediated by the charge $=2 / 3$, scalar LQ $\left(R_{2}^{-1 / 2}\right)$ with $T_{3}=-1 / 2$ and while the other three mediated by vector LQs $\left(U_{1 \mu}, U_{1 \mu}, U_{3 \mu}^{0}\right)$ having $T_{3}=0$ each. The matrix element squared for the 4 diagrams contributing to the $\mathrm{CC}$ s-channel process is

$$
\begin{aligned}
& \left|\mathcal{M}_{L Q}^{s-\operatorname{chann}}\left(\overline{\overline{\nu_{e}}} u \longrightarrow e^{+} b\right)\right|^{2}=\left[\hat{s}\left(\hat{s}-m_{b}^{2}\right)\right]\left[\frac{\left|h_{2 L} h_{2 R}\right|^{2}}{\left(\hat{s}-M_{R_{2}^{-1 / 2}}^{2}\right)^{2}}\right]+\left[4 \hat{u}\left(\hat{u}-m_{b}^{2}\right)\right]\left[\frac{\left|h_{1 L} h_{1 L}\right|^{2}}{\left(\hat{s}-M_{U_{1 \mu}}^{2}\right)^{2}}\right. \\
& \left.+\frac{\left|h_{3 L} h_{3 L}\right|^{2}}{\left(\hat{s}-M_{U_{3 \mu}^{0}}^{2}\right)^{2}}-2 \frac{\left|h_{1 L} h_{3 L}\right|^{2}}{\left(\hat{s}-M_{U_{1 \mu}}^{2}\right)\left(\hat{s}-M_{U_{3 \mu}^{0}}^{2}\right)}\right]+\left[4(\hat{s}+\hat{u})\left(\hat{s}+\hat{u}-m_{b}^{2}\right)\right]\left[\frac{\left|h_{1 L} h_{1 R}\right|^{2}}{\left(\hat{s}-M_{U_{1 \mu}}^{2}\right)^{2}}\right]
\end{aligned}
$$

where, the Mandelstam variables at the parton level are given by $\hat{s}=\left(p_{\overline{\nu_{e}}}+p_{u}\right)^{2}, \hat{t}=\left(p_{\overline{\nu_{e}}}-p_{e^{+}}\right)^{2}$ and $\hat{u}=\left(p_{\overline{\nu_{e}}}-p_{b}\right)^{2}$.

In the u-channel, three diagrams are mediated by the charge $=1 / 3$, scalar LQs $\left(S_{1}, S_{1}, S_{3}^{0}\right)$ with $T_{3}=0$ and one is mediated by a vector $\mathrm{LQ}\left(V_{2 \mu}^{-1 / 2}\right)$ with $T_{3}=-1 / 2$ ( figure $\left.6(\mathrm{~b})\right)$. The matrix element squared for all 4 diagrams contributing to the $\mathrm{CC}$ u-channel process is

$$
\left|\mathcal{M}_{L Q}^{u-\operatorname{chann}}\left(\overline{\nu_{e}} u \longrightarrow e^{+} b\right)\right|^{2}=\left[\hat{u}\left(\hat{u}-m_{b}^{2}\right)\right]\left[\frac{\left|g_{1 L} g_{1 L}\right|^{2}}{\left(\hat{u}-M_{S_{1}}^{2}\right)^{2}}+\frac{\left|g_{1 L} g_{1 R}\right|^{2}}{\left(\hat{u}-M_{S_{1}}^{2}\right)^{2}}+\frac{\left|g_{3 L} g_{3 L}\right|^{2}}{\left(\hat{u}-M_{S_{3}^{0}}^{2}\right)^{2}}\right.
$$




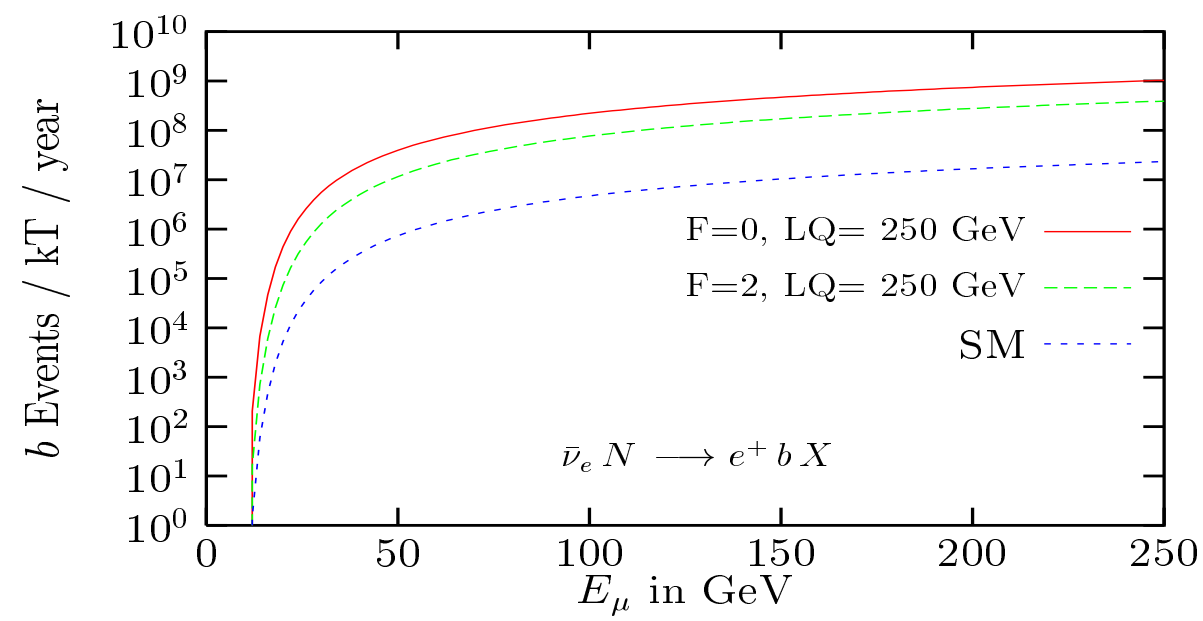

Figure 8: Variation of b-events (from $S M$ and $L Q$ ) for a $1 k T$ detector and $L Q$ mass $250 \mathrm{GeV}$ with muon beam energy for a baseline length 40 meters and sample detector area $0.025 \mathrm{~m}^{2}$

$$
\left.-2 \frac{\left|g_{1 L} g_{3 L}\right|^{2}}{\left(\hat{u}-M_{S_{1}}^{2}\right)\left(\hat{u}-M_{S_{3}^{0}}^{2}\right)}\right]+\left[4(\hat{s}+\hat{u})\left(\hat{s}+\hat{u}-m_{b}^{2}\right)\right]\left[\frac{\left|g_{2 L} g_{2 L}\right|^{2}}{\left(\hat{u}-M_{V_{2 \mu}^{-1 / 2}}^{2}\right)^{2}}\right]
$$

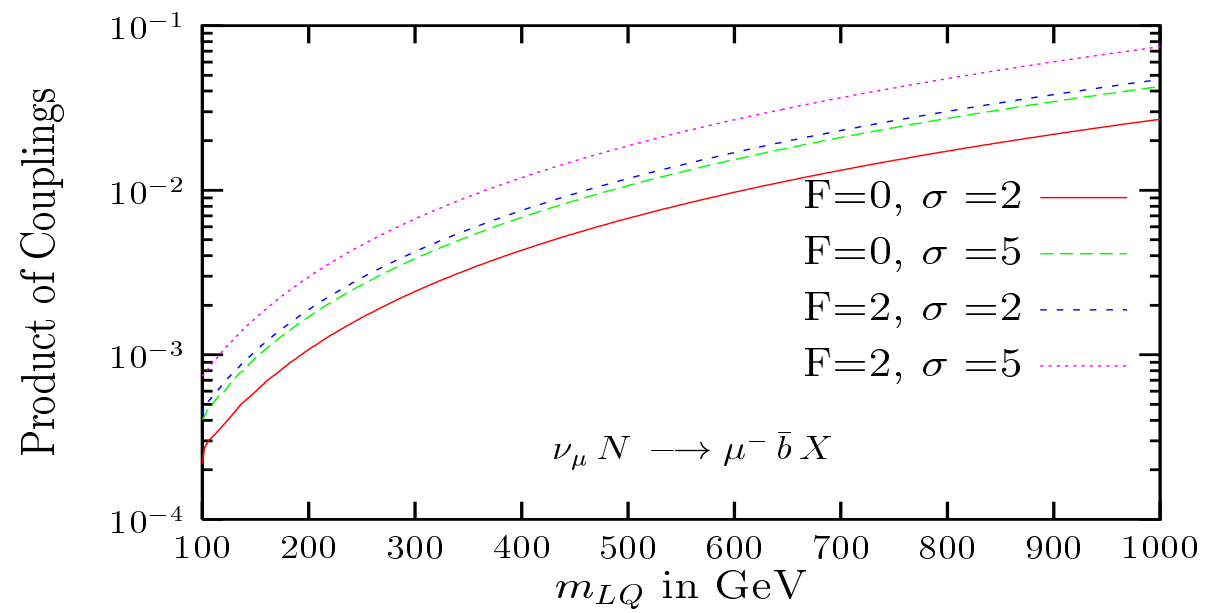

Figure 9: Contour plot for $\bar{b}$ production at $2 \sigma$ and $5 \sigma$ effect for $E_{\mu}=50 \mathrm{GeV}$, baseline length $=40$ meters and sample detector of area $2500 \mathrm{~cm}^{2}$ and mass $1 \mathrm{kT}$.

As discussed in the previous section, we have used the model independent bounds on couplings from [11] and the relevant bounds for the processes listed above are listed in table 1. We extract bounds relevant to $\left(\nu_{\mu} \bar{u}\right)\left(\mu^{-} \bar{b}\right)$ vertex from the bounds for $(21)(23)$ generation of quark-lepton pair, while for the vertex $\left(\bar{\nu}_{e} u\right)\left(e^{+} b\right)$, we use the bounds for the (11)(13) generation indices relevant for the process $\bar{\nu}_{e} u \longrightarrow e^{+} b$. The other inputs to compute the event rates are the same as for the NC diagrams. In figures 7 and 8 , we have plotted the $\bar{b}$ and $b$ event rates as a function of muon beam energy for $\nu_{\mu}-N$ and $\bar{\nu}_{e}-N$ scattering processes respectively. For these processes we have also plotted the SM contribution to $\bar{b}$ and b events. To determine 


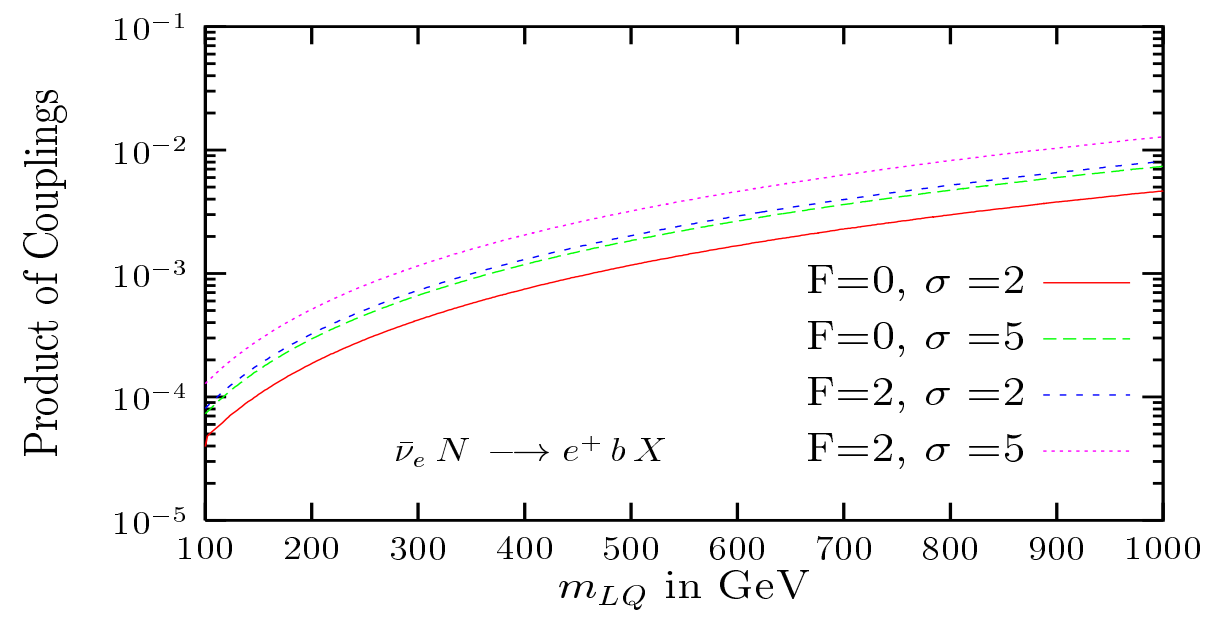

Figure 10: Contour plot for $b$ production at $2 \sigma$ and $5 \sigma$ effect for $E_{\mu}=50$ GeV, baseline length $=40$ meters and sample detector of area $2500 \mathrm{~cm}^{2}$ and mass $1 \mathrm{kT}$.

the allowed range of LQ masses and products of couplings, we have used the criterion that the number of signal events is equal to two or five times the square root of events in the SM. Accepting this requirement of $2 \sigma$ and $5 \sigma$ effect as a sensible discovery criterion the contour in figures 9 and 10 are drawn for a baseline length of $40 \mathrm{~m}$ and thus the non-compliance of these estimates with experimental observation would mean that the region above these curves is ruled out.

\section{Conclusions and Discussion}

Heavy quark $(b, \bar{b})$ production from $\nu_{\mu}-\mathrm{N}$ and $\bar{\nu}_{e}-N$ scattering via both the CC and NC interactions at a NF provides an exciting possibility to detect signals of new physics. This comes about because in these processes the SM contribution is heavily suppressed either due to CKM matrix element or due to interaction of neutrinos with the sea quarks present inside the nucleon. The NC processes in SM are further suppressed as they can take place only at one loop level. We have computed here the $b(\bar{b})$ event rates in theories with LQ and confined ourselves to the near-site experiments where the oscillation effects are negligible. From figure 7 , it is clear that the contribution coming from the SM to the $\bar{b}$ production rate in the CC channel is higher than that of LQ's with $|\mathrm{F}|=0$, while it is lower than the contribution from LQ's with $|\mathrm{F}|=2$ for our choice of the couplings obtained from low energy experiments. We typically find (figure 8 ) that the SM contribution to $b$ production rate is 2 to 3 orders of magnitude smaller than LQ contribution in CC channel even after using the most severe constraints on LQ couplings and masses from low energy FCNC processes. Further the $b$ production rate in the NC channel (figures 3 and 4) is comparable to that for the CC case. We have investigated the region in coupling - mass space for LQ which can provide a reasonable signal for the discovery of new physics involving LQ. It may be noted that this region can be even more restrictive than that implied by the low energy bounds obtained from B meson decays. Also the inclusion of LFV interactions via LQ's could further squeeze the allowed region of LFV Couplings and masses.

Acknowledgment : P.M. acknowledges Council for Scientific and Industrial Research, India 
while A.G. acknowledges the University Grants Commission, India for partial financial support. We also thank SERC, Department of Science \& Technology, New Delhi, for partial financial support.

\section{References}

[1] S. Dutta, R. Gandhi, B. Mukhopadhyaya, Euro. Phys. J.C18, 405 (2000); C. Quigg, (hepph/9803326); S. Geer, Phys. Rev. D57, 6989 (1998); D. Ayres et al., (electronic archive: physics/9911009); A. Cervera et al., (hep-ph/0002108); A. Blondel et al., CERN-EP2000-05; C. Albright et al., (hep-ex/0008064); S. Geer, (hep-ph/0008155).

[2] M.L. Mangano et. al., (hep-ph/0105155).

[3] Y. Fukuda et. al., Phys. Lett. B433, 9 (1998), Phys. Rev. Lett. 81, 1562 (1998); T. Kagita, in proceedings of the XVIIIth International Conference on Neutrino Physics and Astrophysics, Takayama, Japan (June 1998).

[4] L. Wolfenstein, Phys. Rev. D17, 2369 (1978), Phys. Rev. D20, 2634 (1979); S. P. Mikheyev and A. Yu Smirnov, Sov. J. Nucl. Phys.42 (1986) 913.

[5] B. Pontecorvo, Sov. Phys. JETP 26 (1968) 984.

[6] A. Datta, R. Gandhi, B. Mukhopadhayaya, P. Mehta, Phys. Rev. D64, 015011 (2001).

[7] P. Mehta, A. Goyal, S. Dutta, Phys. Lett. B535, 219 (2002).

[8] D. Chakraverty, A. Dutta, B. Mukhopadhayaya, Phys. Lett. B503, 74 (2001).

[9] J. C. Pati and Abdus Salam, Phys. Rev. D10, 275 (1974); O. Shankar, Nucl. Phys. B206, 253 (1982); W. Buchmuller and D. Wyler, Phys. Lett. B177, 377 (1986); W. Buchmuller, R. Ruckl, D. Wyler, Phys. Lett. B191, 442 (1987); P. Langacker, M. Luo, Alfred K Mann, Rev. Mod. Phys. 64, 87 (1992); J. Blumlein and R. Ruckl, Phys. Lett. B304, 337 (1993); M. A. Doncheski and R. W. Robinett, Phys. Rev. D56, 7412 (1997); U. Mahanta, Phys. Rev. D62, 073009 (2000).

[10] See the third and sixth references in [9].

[11] S. Davidson, D. Bailey, B. A. Campbell, Z. Phys. C61, 613 (1994); E. Gabrielli, Phys. Rev. D62, 055009 (2000);

[12] S. Aid et al. Phys. Lett. B353, 578 (1995); Derrick et al., Z. Phys. C73, 613 (1997); C. Adloff et al., (hep-ex/9907002).

[13] H. Lai et al., Phys. Rev. D55, 1280 (1997).

[14] C. Albright et al., (hep-ex/0008064).

[15] K. Abe et. al. (The Belle Collaboration), (hep-ex/0204002), to appear in Phys. Rev. Lett.; B. Aubert et. al. (BABAR Collaboration), (hep-ex/0207080); A. Roodman, (hepex/0112019); V. Halyo, (hep-ex/0207010); J. Nam et. al. (The Belle Collaboration), Int. J. Mod. Phys.A16, Proc. Suppl. 1B, 625 (2001). 